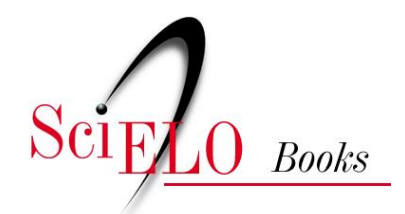

\title{
Políticas públicas e interculturalidad
}

\author{
Amanda Tello Vázquez
}

\section{SciELO Books / SciELO Livros / SciELO Libros}

TELLO VÁZQUEZ, A. Políticas públicas e interculturalidad. In: GARCÉS, F., and BRAVO, R., eds. Interculturalidad. Problemáticas y perspectivas diversas [online]. Quito: Editorial Abya-Yala, 2019, pp. 167-184. ISBN: 978-9978-10-497-2. https://doi.org/10.7476/9789978104972.0006.

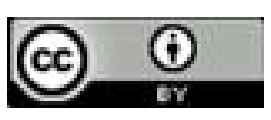

All the contents of this work, except where otherwise noted, is licensed under a Creative Commons Attribution 4.0 $\underline{\text { International license. }}$

Todo o conteúdo deste trabalho, exceto quando houver ressalva, é publicado sob a licença Creative Commons Atribição 4.0.

Todo el contenido de esta obra, excepto donde se indique lo contrario, está bajo licencia de la licencia $\underline{\text { Creative }}$ Commons Reconocimento 4.0. 


\section{Políticas públicas e interculturalidad}

Amanda Tello Vázquez

Universidad Politécnica Salesiana

\section{Introducción}

La interculturalidad ha sido un tema tratado desde varias perspectivas y en áreas específicas; aparece constantemente en los debates públicos para el diseño de políticas públicas y sus instrumentos. En este sentido, se hace necesario revisar las relaciones entre la política pública y lo intercultural. Para el desarrollo del presente trabajo partimos del siguiente planteamiento:

(...) la humanidad genera tantas culturas como grupos sociales existen, cada uno con su propia historia, lógica y significación, convirtiendo a los seres humanos en sujetos portadores de cultura: seres que comparten universos simbólicos que son constantemente socializados a través de procesos educativos. (Tirzo \& Hernández, 2010, p. 13)

Este planteamiento evidencia la importancia que tiene tratar el tema de la diversidad cultural y su expresión en la política pública, convirtiéndola en un objeto de estudio que puede recibir interesantes aportes desde la perspectiva antropológica.

Este artículo no aborda el debate entre multiculturalismo, pluriculturalismo e interculturalidad. Aquí se considera el abordaje por la interculturalidad como concepto integral, que incorpora tanto la existencia, el relacionamiento y el potencial transformador de la diversidad cultural.

Para la revisión de la relación y el análisis entre política pública e interculturalidad, el presente documento se encuentra organizado en tres partes. La primera de ellas desarrolla ciertas referencias teóricas sobre la política pública que permitan, más adelante, establecer los 
mecanismos de relacionamiento entre la interculturalidad y la política pública. En la segunda parte se describe brevemente algunas características de la concepción de interculturalidad que se han desarrollado y que apuntan a brindar elementos para el diálogo con la política pública. La tercera parte procura ser un análisis de los puntos de encuentro, los mecanismos y las consideraciones que debe haber en el trabajo de la política pública, desde una perspectiva de la interculturalidad crítica.

\section{Política pública}

En la literatura especializada existe un sinnúmero de definiciones de política pública. La política pública son líneas de acción determinadas por el Estado por medio de diversos recursos y mecanismos y que buscan resolver un problema que se ha identificado como de interés público. Existen cuatro elementos básicos que permiten identificar una política pública: implicación del gobierno, percepción de problemas, definición de objetivos y proceso. Para el presente artículo, la política pública será entendida como:

Un conjunto conformado por uno o varios objetos colectivos considerados necesarios o deseables, y por medios y acciones (...) con la finalidad de orientar el comportamiento de actores individuales o colectivos para modificar una situación percibida como insatisfactoria o problemática. (Roth, 2010, p. 21)

En este sentido, la política pública se constituye como elemento clave mediante el cual el Estado establece un determinado tipo de relación con la sociedad. Esto a través de dos elementos, el primero de ellos tiene que ver con el contenido de la política y el segundo con proceso de formulación.

¿Qué acerca del contenido de la política? Nos referimos con esto a la forma en que un tema es clasificado como una problemática pública a ser resulta mediante la intervención del Estado. De acuerdo a Roth (2007), la definición de un asunto de política pública es el resultado del "conjunto de las luchas que los actores sociales y políticos libran para imponer una lectura de un problema que sea la más ventajosa posible para sus intereses (entendidos en un sentido amplio)" (p. 58). El autor establece que, de 
manera simplificada, existen dos fuentes para la inscripción de un asunto de política pública "Una es externa al Estado y a sus instituciones —es el modelo de la demanda- y la otra es interna al Estado y a sus instituciones —es el modelo de la 'oferta administrativa'” (p. 62).

Existen distintos modelos de inclusión de problemas en la agenda pública, Garrau (1990) propone cinco modelos que combinan variables y actores entre los que:

Considera a los grupos organizados, la existencia o no de una "demanda social", la presencia de conflictos y de procesos de movilización social, las estrategias de creación de una opinión pública, el papel de la "oferta política" y la mediatización del tema y su dimensión como evento inesperado. (Garrau en Roth, 2007, p. 65)

De la combinación de estos elementos surgen cinco modelos: (i) modelo de movilización: basado en el trabajo de los actores sociales, se caracteriza por "demanda social" fuerte, conflicto entre autoridad y grupos organizados alrededor de la solución a implementar, creación de eventos para atraer la atención de las autoridades y de los medios de comunicación — huelga, marchas, hechos violentos, etc.; (ii) modelo de oferta política: hace referencia al posicionamiento de asuntos en la agenda pública por parte de organizaciones políticas para intentar ganar apoyo o electores, a través de propuestas políticas y tiene como efecto, crear la demanda social; (iii) modelo de mediatización: hace referencia a la influencia de los medios de comunicación en la generación de problemas de política pública, a través de la presentación selectiva de información o su ocultamiento; (iv) modelo de anticipación: este modelo se refiere a las acciones que el aparato público decide emprender para un tema que se analiza como potencialmente problemático, la administración se adelanta a la aparición de un conflicto; y (v) modelo de acción corporativista silencioso: este es un modelo operado desde grupos organizados privilegiados que tienen influencia directa sobre la agenda de las autoridades públicas (Roth, 2007).

Kingdon (en Roth, 2007) propone diferenciar tres factores claves para la inclusión de un problema de acción pública, cuya coincidencia genera lo que denomina ventana política. Los tres factores son los pro- 
blemas, las respuestas o alternativas y la política. La conjunción de estos tres factores posibilita la formulación de la agenda de acción pública:

Cuando un problema encuentra solución adecuada y la política se interesa en él, se abre una ventana política (policy window); entonces las "corrientes" entran en resonancia y así las condiciones están dadas para que la agenda política se haga cargo del problema. (p. 68)

Sobre el proceso de formulación de la política pública, éste se caracteriza por ser diverso y ser un reflejo de las relaciones que el Estado busca establecer con la sociedad. Así, por ejemplo, existen los procesos de formulación top down y bottom up. El primero de ellos hace referencia al modelo en el que la formulación de la política pública es realizada únicamente desde arriba, desde las instancias gubernamentales sin mecanismos de diálogo con actores no estatales, la llamada política de escritorio. En el otro lado tenemos la formulación desde abajo, el modelo bottom up en la que la política pública es el resultado de procesos sociales que logran su manifestación a través de la misma y que garantiza una formulación más amplia y cercana a las necesidades de la sociedad, alcanzando una política más efectiva.

El proceso de formulación de la política pública se da entonces en un constante diálogo de varios actores en la esfera pública. Esta esfera pública está conformada por dos grupos de actores: el sistema institucional - actores estatales - y la sociedad civil. Cada uno de ellos interviene bajo racionalidades específicas que, a su vez, generan una posición y una forma de negociar frente a los asuntos públicos. El sistema institucional debate sobre las decisiones tomadas por los actores políticos, mientras que la sociedad civil procesa las demandas presentadas por la sociedad en su totalidad. Se identifica entonces a los actores estatales y la sociedad civil — reconociendo su amplia diversidad-como dos partes fundamentales para el desarrollo de la política pública.

De ahí que sea importante la perspectiva de la gobernanza. Ésta hace referencia, precisamente, a la relación existente entre el Estado y los actores no estatales en el ejercicio de las políticas públicas; es un mecanismo de regulación de las interacciones entre el Estado y los actores no es- 
tatales, define las reglas de juego, y constituye un instrumento de análisis para comprender el funcionamiento de los sistemas sociales y políticos.

La gobernanza se puede entender como la evolución de las relaciones del Estado y la Sociedad, así como el cambio de rol mismo del Estado. Fontaine (2010) plantea que estas nuevas formas de relacionamiento han generado:

Un cambio en la actividad de gobernar (el gobierno), que pasaría de modo unilateral, basado en la dirección y el control, a un modo multilateral según cual la calidad del sistema depende del reconocimiento mutuo de las capacidades y necesidades de cada uno (Estado y sociedad). (p. 91)

Aquí se ponen de manifiesto las redes de política como "mecanismos de movilización de recursos políticos en temas en los cuales las capacidades y la información necesaria para formular, decidir o implementar programas de acción pública son diseminadas entre muchos actores públicos y privados, algunos con poder de 'veto'" (Roth, 2010, p. 40).

La gobernanza pretende brindar elementos adicionales para un análisis más detallado y profundo de las dinámicas del Estado y la sociedad civil en la definición de los asuntos públicos, así como la participación de cada actor en este tema. Kooiman (2006) plantea:

La gobernanza se logra mediante la creación de estructuras socio-políticas interactivas y procesos que estimulan la comunicación entre los actores involucrados, y la creación de las responsabilidades comunes (...) Existe la necesidad de reestructurar las responsabilidades de gobierno, tareas y actividades, basadas en la diferenciación y la integración de las diversas preocupaciones y los agentes que los representan. (pp. 3-4)

Conforme este planteamiento, existen tres tipos de gobernanza: autogobernanza, co-gobernanza y gobernanza jerárquica. La primera tiene que ver con interacciones sociopolíticas más desordenadas - $\mathrm{o}$ caóticas para utilizar el término exacto del autor-, habla sobre la capacidad de organismos sociales de gobernarse de manera autónoma a través de la posibilidad de construcción de sentidos y significados colectivos que permitan mantener una identidad en común; la segunda - co gobernanza - hace referencia a formas horizontales de gobierno, de 
relaciones entre el Estado y los actores no estatales, siendo una característica importante la autonomía que los actores tienen; el tercer tipo de gobernanza - la gobernanza jerárquica - tiene que ver con una noción del Estado como actor hegemónico, con un alto grado de formalización entre el Estado y los actores no gubernamentales (Kooiman, 2006).

La política pública representa una acción desde el Estado para solventar un problema calificado como público. Esta línea de acción requiere la identificación de mecanismos para su ejecución: los instrumentos de política. Los instrumentos de políticas son "técnicas de gobernanza que, de una manera u otra, envuelven la utilización de la autoridad del Estado o sus limitaciones conscientes" (Howlett, 2005, p. 31). Tanto la definición de la política pública, como sus instrumentos son elementos fundamentales para comprender la acción del Estado y su relación con actores no estatales, marcando el modelo de gobernanza.

Lascoumes y Le Gales (2007 ), plantean dos elementos en relación a los instrumentos de política pública: (i) el instrumento revela la teorización sobre la relación entre el gobierno y la sociedad: todos los instrumentos constituyen formas condensadas de conocimiento de control y de formas de ejercerlo; y, (ii) los instrumentos no son neutrales, producen efectos específicos, independientemente del objetivo perseguido que estructura las políticas públicas bajo su propia lógica (p. 3).

Los instrumentos muestran la teorización de la relación entre el gobernante y los gobernados. El uso de un instrumento tiene un significado específico respecto a la forma de relacionarse del Estado con actores no estatales, así como las vías de ejercicio del poder y los mecanismos de interacción en la esfera pública.

Es importante considerar que los instrumentos de política pública no son herramientas con una neutralidad axiológica perfecta, sino son portadores de valores, llenos de una interpretación de lo social y con nociones precisas del modo de regulación (Lascoumes \& Le Gales, 2007, p. 4). Los instrumentos revelan la manera de relacionamiento del Estado con la sociedad y son una entrada adecuada para comprender los flujos de la gobernanza, entendida como la relación entre todos los actores de la esfera pública. 


\section{La interculturalidad como proyecto político}

La interculturalidad es un término que entró en auge desde principios de la década de los 80 en el ámbito académico y político latinoamericano, con especial énfasis en el ámbito de la educación, aunque su discusión se ha ampliado a diversas ramas de la ciencia — sociología, derecho, comunicación, filosofía, salud etc.

En su comprensión básica, la interculturalidad hace referencia al relacionamiento de distintas culturas que conviven en un mismo espacio. Sin embargo, existen varias consideraciones que se deben plantear respecto al significado y uso de la interculturalidad en el ámbito académico y político, "la interculturalidad encierra un dilema, puede profundizarse y ser progresista e incluso emancipativa, o simplemente un mecanismo de domesticación muy eficiente" (Viaña, 2009, p. 19).

La interculturalidad es un concepto complejo que puede fluctuar de una comprensión básica que permita conservar el statu quo, pretendiendo, mediante medidas específicas solventar las problemáticas de la relación entre diversos y las crecientes demandas sociales respecto a modelos más inclusivos; hacia una comprensión compleja en la que la interculturalidad se convierte en un elemento efectivamente transformador de las estructuras sociales.

Existen varias formas de entender y del uso de la interculturalidad. Walsh (2010) propone tres perspectivas. La primera de ellas, la relacional, mencionada previamente, hace referencia al intercambio y contacto entre culturas, saberes, tradiciones, prácticas culturales distintas, relaciones que se pueden dar en condición de igualdad o desigualdad. Esta forma de compresión de la interculturalidad simplifica el encuentro entre culturas, pues oculta los conflictos y las luchas de poder inherentes al relacionamiento entre culturas. Esta es la manera más común en la que se piensa la interculturalidad, desde diversos espacios sociales y estatales. La interculturalidad como relacionamiento entre diversos es el punto de partida de reflexiones académicas y políticas que deja de lado los elementos más problemáticos de este encuentro.

La segunda perspectiva se denomina funcional, plantea el reconocimiento de la diversidad y diferencia cultural, para la inclusión de la 
misma al interior de la estructura social dominante. De esta manera, el diálogo, la convivencia y la tolerancia son funcionales al sistema existente y no atacan las causas de la asimetría y desigualdad social, pues solo proponen subsumir una cultura a otra dominante. La interculturalidad entendida como la inclusión de una cultura en el sistema dominante, se puede calificar, más bien, como un ejercicio de multiculturalismo, en el que se reconoce la diferencia y diversidad, pero se elimina las consideraciones estructurales del reconocimiento de esta diversidad y de su inclusión, como un ejercicio de dominación. En esta línea, Tubino (en Sinnigen, 2013) describe el multiculturalismo como una acción afirmativa de mutuo respeto y tolerancia, en contraposición con la interculturalidad como una acción transformativa que va más allá del respeto y la tolerancia y apunta hacia un diálogo destinado a lograr la igualdad cultural, económica y política con la diversidad cultural.

La tercera, la interculturalidad crítica, hace referencia a la comprensión de ésta como un proceso que apela al cambio de las estructuras coloniales. En este caso la interculturalidad se entiende como:

Una herramienta, como un proceso y proyecto que se construye desde la gente $-\mathrm{y}$ como demanda de la subalternidad- (...). Apuntala y requiere la transformación de las estructuras, instituciones y relaciones sociales, y la construcción de condiciones de estar, ser, pensar, conocer, aprender, sentir y vivir distintas. (Walsh, 2010, p. 78)

La interculturalidad crítica se convierte en la forma más integral de entender la relación entre culturas. El relacionamiento entre diversos no se basa en el reconocimiento y la inclusión en un sistema hegemónico, se trata de cuestionar los cimientos del sistema:

La interculturalidad deberá ser concebida como un proyecto en construcción de un proceso de igualación real de todas las personas, alejado de las siempre presentes relaciones de poder y dominación que subsumen la capacidad creativa y refuerzan la colonialidad. (Chávez, s.f, p. 145)

Siguiendo esta última acepción, es evidente que la interculturalidad es el producto de un proceso político que busca transformar las bases del sistema inequitativo, en busca de una sociedad más justa y reconocedora de la diversidad cultural como un elemento de cambio estructural. 
En este sentido, la participación de actores no estatales es fundamental, pues es a través de la misma que se posibilitará el reconocimiento a la diversidad de manera íntegra. Como lo menciona Guerrero (2010) "es necesario no olvidar el carácter social y político que ésta tiene, pues es resultante de prácticas, acciones y luchas sociales concretas y conscientes que llevan adelante sujetos sociales, políticos e históricos igualmente concretos" (p. 461).

\section{¿Políticas públicas interculturales?}

Como se ha mencionado previamente, para que exista una política pública, debe existir un problema considerado de interés público que debe, de una u otra manera, ser atendido por el Estado a través de una serie de instrumentos que permitan alcanzar el objetivo de política. Para que un asunto sea identificado como de interés público se hace necesaria la conjunción de al menos tres elementos mencionados anteriormente: un problema, una respuesta o alternativa y el interés político. Si estos elementos se encuentran se abre una ventana política.

La interculturalidad, en sus diversas acepciones, se ha convertido en un asunto de política pública pues conjuga los tres elementos planteados anteriormente. Se constituye como un problema en tanto los actores sociales demandan la consideración de la diversidad cultural en todas las áreas de la vida social; se proponen respuestas o alternativas para su solución a través de políticas manifestadas en programas y proyectos de diversa índole que apuntan a satisfacer la demanda social. Y para el tercer elemento, la política, la interculturalidad le resulta atractiva como fuente de capitalización de apoyo a determinado proyecto político.

Es importante partir de que la interculturalidad ha sido un tema posicionado en la agenda pública principalmente por los movimientos indígenas de la región, en demanda a la construcción de sociedades más equitativas y justas. Esta forma de constituirse en asunto público se puede clasificar en el modelo de movilización de Roth (2007). El objeto toma interés general a partir de una demanda social, en la que existe conflicto entre la autoridad y los grupos de interés para la identificación de las soluciones a implementarse y se basa en un amplio manejo mediático que introduce la temática en el debate público. 
Como se mencionó previamente, los instrumentos de política pública o las formas de intervención no son neutrales, sino son portadores de valores y responden a una interpretación particular de lo social. Las formas de intervención estatal sobre la problemática de la diversidad cultural y la interculturalidad son innumerables y su elección dependerá de la intencionalidad de la intervención estatal, se busca generar una interculturalidad de carácter relacional o funcional se determinará el uso de ciertos instrumentos, mientras que, si se busca la construcción de una interculturalidad crítica, otros serán los métodos prioritarios.

La inclusión de la interculturalidad en la agenda pública puede presentarse como una serie de declaraciones, sin una expresión concreta. Es decir, se muestra como un principio que reconoce la diversidad y dicta principios de tolerancia y respeto, pero no se materializa en acciones específicas. Se trata de declaraciones:

Inofensivas, líricas, en alguna parte de la Constitución Política del Estado, planteando la constatación de lo pluriétnico y multicultural de los países y Estados, constatación que no lleva a ningún cambio estructural ni del Estado ni de la sociedad en la perspectiva de su interculturalización profunda. (Viaña, 2009, p. 27)

Por ejemplo, la Constitución del Ecuador contempla una serie de enunciados alrededor de la plurinacionalidad y la interculturalidad, convirtiendo a este cuerpo normativo en altamente progresista en el nivel discursivo. Al respecto, García (2009) resalta cuatro elementos contenidos en el texto constitucional, en los que se podría apreciar un intento de interculturalización: (i) conocimiento y ciencia: se reconoce que el conocimiento no es singular, los saberes ancestrales tienen el estatus de conocimiento lo que implica la incorporación de otras formas de conocimiento con igual importancia que la Occidental; (ii) los derechos de la naturaleza: a través de esto se integra la forma de comprender la naturaleza desde la filosofía y cosmovisión indígena en la que el ser humano es un elemento más y no una ser externo superior que únicamente usufructúa de la misma; (iii) el sistema jurídico: se reconoce la justicia indígena, un reconocimiento que puede convertirse en más que un pluralismo jurídico, formulando una relación de coordinación 
y cooperación entre la jurisdicción ordinaria y la indígena; (iv) sumak kawsay o buen vivir: esto permitiría concebir y agenciar la vida desde otra perspectiva, considerando la posibilidad de un nuevo contrato social enraizado en la convivencia ética entre seres humanos y su entorno, alejándose del individualismo que caracteriza al modelo de la política neoliberal (pp. 23-25).

Otra forma de intervención estatal sobre la interculturalidad es la formulación de políticas públicas particularizadas para todas las diversidades. En este sentido, se formulan políticas específicas de educación, salud, justicia, territorio, etc. a partir del reconocimiento de la diversidad, que buscan atender las particularidades culturales pero que pueden constituirse también en mecanismos exclusionarios, generando sistemas paralelos de atención a los grupos culturales diferentes, sin promover el relacionamiento y conocimiento entre diversos.

En el caso ecuatoriano, esto se evidencia en el sistema educativo intercultural bilingüe que constituía, hasta la emisión de la Ley Orgánica de Educación Intercultural, un sistema paralelo de atención específica para los pueblos y nacionalidades. A partir de la emisión de la LOEI este se fusiona al sistema nacional, dando paso a la creación del sistema de educación intercultural que en la actualidad se encuentra vigente.

Una tercera forma de abordar la interculturalidad es el enfoque transversal, en el que la interculturalidad cruza todas las políticas estatales procurando la integración de la problemática en todos los ámbitos de interés público. Como principio, la inclusión de la interculturalidad como un eje transversal potenciaría su capacidad crítica y transformadora, pues se integra en todas las esferas de acción estatal y aportaría a la construcción de la interculturalidad crítica como ideal de convivencia en diversidad. Sin embargo, en esta forma de gestión, se corre el riesgo de que las intervenciones pierdan de vista los elementos claves de diversidad cultural que deben ser considerados y se transforme en una serie de elementos funcionales que pierdan el potencial político de transformación, o se conviertan nuevamente en enunciados sin acciones específicas que respalden su cumplimiento.

Como uno de los mecanismos de transversalización adoptados en el país, tenemos a los Consejos Nacionales para la Igualdad, instancias 
creadas en la Constitución y regidas por la Ley Orgánica de los Consejos Nacionales para la Igualdad. Estas son instancias de composición mixta - actores estatales y no estatales - que tienen como finalidad participar en todo el ciclo de la política, asegurando la incorporación de todas las temáticas transversales en las políticas sectoriales. Este mecanismo se aplica desde el 2014, año en el que se expidió la ley que reguló su funcionamiento de acuerdo a los principios establecidos en la Constitución.

Como se evidencia, el reconocimiento de la interculturalidad como tema de interés público a ser atendido por el Estado genera un grado de formalización e institucionalización del mismo y se sujeta a sus limitaciones y ventajas. De esto se derivan algunas inquietudes:

¿Qué es lo que el interculturalismo latinoamericano gana y pierde al convertirse en parte del discurso oficial del Estado? ¿Los discursos socialmente críticos tienden a ser funcionales y técnicos cuando se incorporan al aparato del Estado? (...) ¿Qué debemos hacer para que los conceptos y prácticas interculturales ganen terreno en el discurso oficial sin perder su potencial crítico y liberador? ¿Puede el discurso oficial ser funcional y crítico al mismo tiempo? ¿Por qué los discursos y prácticas propuestos pierden su capacidad creativa y crítica cuando se convierten en discursos y prácticas institucionalizadas? ¿Qué pasa con los discursos y prácticas interculturales cuando han dejado de ser propuestas y se han convertido en discursos y prácticas institucionalizadas? (Tubino, 2005, p. 84)

La incorporación de la interculturalidad como parte del discurso estatal puede hacer que ella se desvincule de su origen crítico y liberador, transformándose en un elemento funcional para la conservación de las condiciones actuales de desigualdad y exclusión. Formas básicas de atención a la diversidad cultural pueden aplacar el problema creciente que tiene salida en la consolidación de un modelo intercultural crítico. Así, la interculturalidad se incluye a nivel enunciativo en el discurso político de los actores de la arena pública, pero es una enunciación vacía que no está seguida por medidas concretas para su construcción real:

$\mathrm{Al}$ ser traducidas al lenguaje técnico y jurídico de los Estados, se domestica totalmente la propuesta y se transforma en inofensiva y funcional al neoliberalismo (...) al ser incorporada a las estructuras y los aparatos del 
Estado, quede despojada de sus características críticas y emancipativas. (Viaña, 2009, pp. 19-20)

Entonces, ¿cómo encontrar el equilibrio para que la consideración de la interculturalidad en la agenda estatal, no implique que su sentido crítico se pierda? Lo que se requiere es un diálogo permanente entre los actores sociales y el Estado en el que se asegure que los temas que se quieren posicionar, su proceso de formalización no termine convirtiéndolas en mecanismos de represión cultural, siendo funcionales al sistema hegemónico en el que las diversidades se incluyen como parte de la cultura dominante. Es decir, los actores no estatales deben estar en la capacidad de incidir y renovar las discusiones acerca de las formas y metas que se requieren para la construcción de una verdadera interculturalidad crítica. Como lo menciona Huerta (2016):

La construcción cotidiana de las políticas públicas se convierte en una arena política en tanto se pone en juego un proyecto ideológico de dominación, así como el acceso a recursos simbólicos y materiales. En este sentido, forman parte de negociaciones, acuerdos y conflictos en los que se legitiman determinado tipo de relaciones sociales, la formación de sujetos políticos y el establecimiento de un futuro deseable. (p. 129)

La intervención permanente de los actores no estatales en el debate político y la formulación de la política pública es la única manera en la que el carácter crítico de la interculturalidad se puede mantener, pese a su inclusión como un asunto de atención estatal.

Queda en evidencia la importancia que tiene la sociedad civil como actor fundamental de la implementación de políticas e instrumentos que promuevan la interculturalidad crítica. La capacidad de agencia de los actores no estatales es fundamental para generar el dinamismo que la inclusión de esta temática requiere en el aparato estatal.

Cabe aquí pensar qué tipo de gobernanza se debe generar para poder apuntar a la construcción de la interculturalidad crítica. Como se mencionó en la primera parte de este artículo, hay tres modelos de gobernanza que se determinan a través de las formas de relación y los roles del Estado y la sociedad. Para el caso que nos ocupa, el modelo que aportaría a la generación de un modelo de interculturalidad crítica es la 
llamada co-gobernanza en la que la forma de gobierno se basa en relaciones horizontales, lo que permitiría una influencia permanente de la sociedad civil en el diseño de políticas vinculadas a la interculturalidad.

Existe la necesidad de revisar las responsabilidades del Gobierno y de los actores sociales. En el modelo de co-gobernanza, la integración de los agentes promotores de la construcción de una interculturalidad crítica en la gestión de la política pública permitirá generar no solamente que sus principios se aseguren, sino también que se asuman tareas y actividades específicas que aporten a la construcción de un sistema más equitativo y justo, como lo demanda los principios de la interculturalidad crítica.

Es importante también atender a los instrumentos que se propongan implementar en el marco de las políticas interculturales, pues estos revelan el enfoque que el Estado proyecta sobre la interculturalidad. En tal sentido, se pueden plantear medidas integracionistas que no cuestionen las estructuras desiguales y que por tanto se basen en una interculturalidad funcional. Este modelo apunta a un proceso etnofágico (Viaña, 2009) que implica una inclusión subordinada al modelo dominante, se reconoce la diversidad, pero dentro de la lógica, dinámica, las instituciones y las estructuras dominantes.

Por otro lado, se pueden pensar en instrumentos que revisen toda la estructura hegemónica, promoviendo un cambio desde las estructuras para la construcción de una sociedad intercultural, en su comprensión amplia. Desde esta perspectiva "hay que apuntar a una interculturalidad como un insumo básico de un proyecto de transformación societal. Hay que pensar la interculturalidad en su sentido más profundo, como elemento de potenciamiento y catalizador de cambios estructurales" (Viaña, 2009, p. 35).

Es necesario recalcar aquí la importancia que tienen los espacios locales, la política local como el escenario preciso para la generación de políticas e instrumentos que apunten al fortalecimiento de modelos interculturales críticos. Los espacios de deliberación de los gobiernos locales constituyen herramientas fundamentales para el empoderamiento de los actores no estatales y la conservación del espíritu crítico de las demandas que apuntan a la transformación de los cimientos del modelo político y social dominante. En este sentido, Torres (2009) define como la gestión local intercultural como: 
El sistema de decisiones y acciones por el cual los gobiernos autónomos descentralizados transforman las expectativas y objetivos interculturales de los pueblos, nacionalidades y comunidades indígenas, afroecuatorianas y montubias en intervenciones públicas que beneficien a la totalidad de la población (...) se refiere a la elección de opciones sobre el conjunto de factores humanos, técnicos, económicos y políticos que coadyuven el más eficaz y eficiente desempeño de la administración gubernamental autónoma descentralizada, contando con la participación de las poblaciones directamente afectadas para la consecución de los resultados interculturales. (p. 73)

Se deriva de esto que la gestión local es el escenario idóneo para la gestión y consolidación de la interculturalidad desde una perspectiva crítica, que permita construir políticas e instrumentos aplicables, en posterior, al debate nacional.

Torres (2009) afirma, así mismo, que deben existir cuatro niveles complementarios para una gestión local intercultural exitosa: (i) una agenda de prioridades interculturales, es decir, lo que se mencionó previamente sobre la problematización de determinada temática para colocarla como problema público a resolverse bajo la intervención del Estado, en relación con los actores no estatales; (ii) una organización institucional adecuada y flexible para la implementación de políticas e instrumentos y que genere espacios de participación para las comunidades indígenas, afro, montubias y mestizas; (iii) personal calificado, sensibilizado para la implementación de las acciones tendientes al fortalecimiento de la interculturalidad crítica; (iv) organizaciones y movimientos sociales activos, movilizadas para el fortalecimiento del modelo de co-gobernanza, mencionado previamente.

La formulación y gestión de la política intercultural, nacional y local, desde la perspectiva crítica debe apuntar siempre a un cambio integral:

Debe promover un sentido político diferente de participación de las diversidades sociales, que haga posible que estas construyan procesos de autogestión económica, autonomía política y revitalización de su identidad, de su memoria colectiva y su cultura, que respondan a sus propios horizontes históricos. (Guerrero, 2010, p. 476) 
La convivencia entre las lógicas estatales y las diversidades es el reto más importante en el ciclo de la política pública intercultural y es lo que genera la necesidad de modificar o crear nuevos mecanismos de diálogo que abran los canales necesarios para la construcción de una interculturalidad crítica.

\section{A modo de conclusión}

Los aportes que se pueden brindar desde la Antropología para el estudio de la política pública es la posibilidad de verlas "como un objeto cultural que puede ser analizado desde la interacción (prácticas culturales) como desde el ámbito ideacional (significados), siempre que se atiendan los procesos" (Huerta, 2016, p. 127). Ver la política pública como un proceso permite decodificar las dinámicas que se establecen entre los actores estatales y no estatales, las correlaciones de fuerzas y los espacios de diálogo en los que se pueda promover la construcción de un modelo de interculturalidad crítico que aporte a la revisión de las estructuras sociales, haciendo de la diversidad una fuerza movilizadora.

Las reflexiones que se presentaron apuntan a contribuir al diálogo entre la teoría de políticas públicas con las discusiones desarrolladas alrededor de la interculturalidad, buscando aportar a la respuesta a ¿cómo lograr que la incorporación de la interculturalidad como un asunto de política pública, no implique que ésta pierda su naturaleza transformadora?

Conjugar el componente transformador que la interculturalidad debe tener con los procesos de la gestión estatal es un reto. El diseño y la implementación de la política pública que busquen responder a las demandas de la interculturalidad, desde una perspectiva crítica, debe presentarse como participativa y flexible. Uno de los puntos clave para lograr este diálogo transformador es la innovación constante de los debates, los actores y los recursos que participan de este proceso. Esta permanente innovación permitiría que la naturaleza crítica y transformadora de la interculturalidad no se vea aplacada por las dinámicas burocráticas.

Todos los esfuerzos, tanto del aparato estatal como de la sociedad civil, como lo menciona Guerrero (2010), deben promover que las políticas públicas interculturales generen nuevos contextos sociales en los que 
el diálogo entre pares permita construir nuevas modernidades, "frente al sentido excluyente y hegemónico de la modernidad occidental marcada por la colonialidad, emergen otras formas de modernidad alternativas o alternativas a lo modernidad, pensadas desde políticas del lugar”. (p. 472)

\section{Bibliografía}

Chávez, D. (s.f). Construcciones teóricas para descolonizar la colonialidad del saber y la construcción de interculturalidad crítica. Integra Educativa, III(1), 133-147.

Fontaine, G. (2010). Petropolítica. Una teoría de la gobernanza energética. Quito: FLACSO.

García, F. (2009). La interculturalidad y las políticas públicas. En Lineamientos para la construcción de políticas públicas interculturales. Memoria del Seminario de Políticas Públicas e Interculturalidad (pp. 16-31). Quito: Ministerio Coordinador de Patrimonio.

Guerrero, P. (2010). Corazonar. Una antropología comprometida con la vida. Quito: Abya-Yala.

Howlett, M. (2005). What is a policy instrument? Policy Tools, Policy Mixes and Policy Implementation. En M. M. Pear Eliadis, Designing Government. From Instruments to Governance. Canadá: McGill-Queen's University Press.

Huerta, M. G. (2016). Procesos interculturales y contrucciones sociales: apuntes para una antropología de las políticas públicas. Iberoamérica Social: revista-red de estudios sociales, VI.

Kooiman, J. (2006). Governing as Governance. Londres: SAGE Publications.

Lascoumes, P., \& Le Gales, P. (2007). Introduction: Understanding Publico Policy through its Instruments. From the Nature of Instruments to the Sociology of Public Policy Instrumentation. Governance: A international Journal of Policy, Administration and Institutions, 20.

Roth, A.-N. (2007). Políticas públicas. Formulación, implementación y evaluación. Bogotá: Ediciones Aurora.

(2010). Enfoques para el análisis de políticas públicas. Bogotá: Universidad Nacional de Colombia.

Sinnigen, J. (2013) Introducción: Intercultural Practices in Latin American Nation States. Journal of Intercultural Studies, 34(5), 604-619. 
Tirzo, J., \& Hernández, J. (2010). Relaciones interculturales, interculturalidad y multiculturalismo; teorías, conceptos, actores y referencias. Cuicuilco.

Torres, V. H. (2009). La acción pública intercultural. Quito: Abya-Yala.

Tubino, F. (2005). La praxis de la interculturalidad en los Estados nacionales latinoamericanos. Cuadernos Interculturales, 5.

Viaña, J. (2009). La interculturalidad como herramienta de emancipación. Hacia una redefinición de la interculturalidad y de sus usos estatales. La Paz: Campo Iris.

Walsh, C. (2010). Interculturalidad crítica y educación Intercultural . En J. Viaña, L. Tapia, \& C. Walsh, Construyendo interculturalidad crítica. La Paz: Instituto Internacional de Integración del Convenio Andrés Bello. 